\title{
The Lattices of Six-Dimensional Euclidean Space
}

\author{
By W. Plesken* and W. Hanrath
}

\begin{abstract}
The lattices of full rank of the six-dimensional Euclidean space are classified according to their automorphism groups (Bravais classification). We find 826 types of such lattices.
\end{abstract}

I. Introduction. A lattice $L$ in an Euclidean vector space $E$ is given by the $\mathbf{Z}$-span of some basis of $E$. Its automorphism group $\operatorname{Aut}(L)$ consists of all orthogonal transformations of $E$ which map $L$ onto itself. Finally, two lattices $L_{1}$ and $L_{2}$ in $E$ are called (Bravais-) equivalent, if there is a linear (not necessarily orthogonal) transformation $\varphi: E \rightarrow E$ with $\varphi\left(L_{1}\right)=L_{2}$ and $\varphi^{-1} \operatorname{Aut}\left(L_{2}\right) \varphi=\operatorname{Aut}\left(L_{1}\right)$. Translating these concepts into the language of matrices by choosing Z-bases of the lattices, shows that the Bravais types of lattices just defined are in 1-1-correspondence with the conjugacy classes of certain finite subgroups of $G L_{n}(\mathbf{Z})$, namely of the Bravais groups, cf., e.g., [1], defined as

$$
B(S)=\left\{g \in G L_{n}(\mathbf{Z}) \mid g^{t r} F g=F \text { for all } F \in S\right\}
$$

for some set $S$ of symmetric real $n \times n$-matrices containing at least one positive definite matrix (which guarantees the finiteness of $B(S)$ ). Dually one assigns to each finite subgroup $G$ of $G L_{n}(\mathbf{Z})$ the vector space

$$
S(G)=\left\{F \in \mathbf{R}^{n \times n} \mid F^{t r}=F, g^{t r} F g=F \text { for all } g \in G\right\} .
$$

The lattices of a fixed Bravais type corresponding to a conjugacy class $\mathfrak{B}$ of Bravais groups can most conveniently be described by $S(B)$ for some representative $B \in \mathfrak{B}$ : A lattice $L$ belongs to the Bravais type under consideration if and only if the Gram matrix $F$ of the bilinear form on $E$ with respect to some $\mathbf{Z}$-basis of $L$ belongs to $S(B)$ and satisfies $B(\{F\})=B$. (Note, the positive definite matrices $F$ in $S(B)$ with $B(\{F\}) \neq B$, i.e. $B(\{F\}) \supsetneqq B$, form a subset of measure zero of $S(B)$.) Therefore, we give our classification by listing representatives $B$ for the conjugacy classes of the Bravais groups in $G L_{6}(\mathbf{Z})$ and $\mathbf{R}$-bases for the corresponding $S(B)$. These data together with additional information explained in Section IV can be found on the microfiches at the end of this issue. The results were obtained by extensive calculations on the Cyber 175 of the Rechenzentrum der RWTH, Aachen, by methods mainly developed in [10].

As for the history of the subject and the connections with mathematical crystallography, the reader is referred to the survey article [11] or to [1]. Here we only mention

Received October 18, 1983.

1980 Mathematics Subject Classification. Primary 20H15, 10E45, $20 \mathrm{C} 10$.

Key words and phrases. Bravais lattices, integral matrix groups.

* Supported by the Deutsche Forschungsgemeinschaft. 
that there are $1,5,14,64,189$, and 826 Bravais types of lattices in 1, 2, 3, 4, 5 resp. 6-dimensional Euclidean space, and that our methods are based on integral representation theory of finite groups rather than on the geometry of quadratic forms cultivated by the Russian school, cf. e.g. [15]. Because of the classification of irreducible Bravais groups of degree 6 in [13] and [10] we may confine ourselves to reducible groups here (which, however, form 806 of the 826 classes). At the end of Section $\mathrm{V}$ we comment on the combinatorial aspects of the geometry of quadratic forms.

II. Methods, Review of Known Results, Comments on the Computation. Because of two basic ideas, the classification of 6- (and probably also 7-) dimensional lattices comes within the reach of our computational possibilities. The first is the concept of the Bravais group $B(G):=B(S(G))$ of a finite unimodular group $G$ due to $\mathrm{H}$. Zassenhaus, cf. [1]. The second is the centering algorithm, cf. [7], [13], also [9], which is an effective procedure to split up the Q-class of finite unimodular groups into $\mathbf{Z}$-classes by producing sublattices of the natural lattices $\mathbf{Z}^{n \times 1}$ on which the groups under consideration act. (Terminology: $G, H \leqslant G L_{n}(\mathbf{Z})$ lie in the same Q-class (Z-class) or are called Q-equivalent (Z-equivalent), in symbols $G \sim{ }_{\mathrm{Q}} H\left(G \sim{ }_{\mathrm{Z}} H\right)$, if $G$ and $H$ are conjugate under $G L_{n}(\mathbf{Q})\left(G L_{n}(\mathbf{Z})\right)$. Note, $\mathbf{Q}$-classes are well understood by classical representation theory.) As discussed in great detail in [6], the Bravais classification of finite unimodular groups is highly incompatible with $\mathbf{Q}$-equivalence. To utilize the convenient Q-classes nevertheless, we define Bravais-minimal groups: A finite unimodular group $G$ is called Bravais-minimal, if $B(H) \neq B(G)$ (and hence $B(H) \lesseqgtr B(G)$ ) for every proper subgroup $H$ of $G$. Note, being Bravais-minimal is a property of $\mathbf{Q}$-classes, whereas being a Bravais group is only a property of $\mathbf{Z}$-classes. The concepts discussed so far suggest to proceed in a given dimension $n$ roughly as follows:

(i) Find the Bravais-minimal subgroups of $G L_{n}(\mathbf{Z})$ up to Q-equivalence;

(ii) Split the Q-classes of (i) into Z-classes, find the associated Bravais groups, and decide which Bravais groups are $\mathbf{Z}$-equivalent.

To use at least some information available from lower dimensions for (most) reducible Bravais groups, the above strategy is slightly modified and refined by the use of almost decomposable Bravais groups introduced in [10].

(I.1) Definition. Let $G \leqslant G L_{n}(\mathbf{Z})$ be finite (and reducible), denote the natural $\mathbf{Z} G$-lattice $\mathbf{Z}^{n \times 1}$ by $L$.

(i) $\tilde{L}=\tilde{L}(G):=\oplus_{e} e L$, where $e$ runs through the set of primitive central idempotents of the rational group ring $\mathbf{Q} G$. (Note, $\tilde{L}$ is a $\mathbf{Z} G$-lattice in $\mathbf{Q}^{n \times 1}$ containing $L$ with finite index; note also $e \mathbf{Q} L$ is a homogeneous component of the action of $G$ on $\mathbf{Q} L$.)

(ii) $\tilde{G}$ is the Z-class of finite unimodular groups defined by the action of $G$ on $\tilde{L}$. (Note, $\tilde{G}$ and $G$ lie in the same Q-class.)

(iii) $P(G)=\{B(H) \mid H \in \tilde{G}\}$ is called the Z-class of almost decomposable Bravais groups associated with $G$.

The most important properties of this construction are the following: If $G_{1}, G_{2}$ are finite unimodular groups with $B\left(G_{1}\right) \sim{ }_{\mathrm{z}} B\left(G_{2}\right)$, then $P\left(G_{1}\right)=P\left(G_{2}\right)$. If $B$ is a Bravais group, then (any) $X \in P(B)$ has a subgroup $Y$ with $Y \sim{ }_{\mathrm{Q}} B$ and $B(Y)=X$. 
Here is the outline of the procedure how we determine the $\mathbf{Z}$-classes of all Bravais groups defining the same $\mathbf{Z}$-class $P$ of almost decomposable Bravais groups with given representative $B \leqslant G L_{n}(\mathbf{Z})$, cf. also [10].

(i) Find $\operatorname{Min}(B)=\{G \leqslant B \mid B(G)=B, G$ Bravais-minimal $\}$.

(ii) Compute for $L=\mathbf{Z}^{n \times 1}$

$$
\operatorname{Cen}(L)=\bigcup_{G \in \operatorname{Min}(B)} \operatorname{Cen}_{G}(L) \text { with } \operatorname{Cen}_{G}(L)=\left\{M \mid M \leqslant_{\mathbf{z} G} L, \tilde{M}(G)=L\right\} \text {. }
$$

(iii) Find a finite set of generators for the normalizer $N=N_{G L_{n}(\mathbf{Z})}(B)$ of $B$ in $G L_{n}(\mathbf{Z})$ and find representatives $M$ of the orbits of $N$ on $\operatorname{Cen}(L)$ (under the obvious action).

(iv) Choose a Z-basis for each $M$ in (iii) and compute the action of $H(M)=\{h \in$ $B \mid h M \leqslant M\}$ on $M$ with respect to this basis. The resulting matrix groups form a set of representatives of the $\mathbf{Z}$-classes of all Bravais groups $B^{\prime}$ with $P\left(B^{\prime}\right)=P$.

We now comment on how we performed these steps for $n=6$. There is, first of all, step 0 , namely to find the Z Z-classes of all almost decomposable Bravais groups. This was done by hand, by means of the family symbol, which we discuss at the beginning of Section IV.

Step (i) is the most time consuming step since we did it by hand. There is, however, the possibility of performing this step completely by machine, for instance by using the Aachen subgroup program in Cayley system; cf., e.g., [5], [3]. But in this case huge data sets as input for step (ii) would be generated. The point is that some theoretical insight shows in almost every particular case that a proper subset $\widetilde{\operatorname{Min}}(B)$ of $\operatorname{Min}(B)$ suffices to obtain a set

$$
\widetilde{\operatorname{Cen}}(L)=\bigcup_{G \in \widehat{\operatorname{Min}}(B)} \operatorname{Cen}_{G}(L)
$$

with the property that each $N$-orbit on $\operatorname{Cen}(L)$ (to be computed in step (iii)) has a representative in $\widetilde{\operatorname{Cen}}(L)$. Therefore it is sufficient to compute representatives for the orbits under the conjugation action of $N$ on $\operatorname{Min}(B)$. Moreover some detailed knowledge of the constituent groups of $B$ often admits predictions on $\operatorname{Cen}_{G}(L)$ for certain $G \in \operatorname{Min}(B)$, for instance to the effect that some of the $\operatorname{Cen}_{G}(L)$ do not yield any new $N$-orbit in $\operatorname{Cen}(L)$. This important point will be demonstrated in Section III by the most instructive and simplest case of Bravais groups with two constituent groups. This kind of consideration enabled us to choose a subset $\widehat{\operatorname{Min}}(B)$ containing only 2 groups on the average, and at most 12 groups (cf. family XLVI in Section IV). In some cases we replaced $\operatorname{Min}(B)$ by a small set of subgroups $H$ of $B$ with $B(H) \lesseqgtr B$. In such a case it might happen that the union of $N$-orbits on the resulting lattices (to be computed in step (ii)) properly contains $\operatorname{Cen}(L)$. This, however, is easily checked in step (iv) (namely $M \in \operatorname{Cen}(L)$ iff $B(H(M)$ ) $=B$ ).

Step (ii) was done by machine computation with the input from step (i) by using a modified version of the implementation of the centering algorithm used in [13], [14]. This modified algorithm uses as input generators of the group $G$ coming from step (i) and the modular constitutents of the natural representation $\Delta$ modulo the prime divisors of the group order $|G|$. The output is the finite set $\operatorname{Cen}_{G}(L)$ in the form of bases for the sublattices. As in [13], [14] the sublattices are computed successively starting with $L$ by computing the kernels of epimorphism of the already obtained 
lattices onto simple $\mathbf{Z} / p \mathbf{Z} G$-modules for prime divisors $p$ of $|G|$. For each new lattice it is checked whether it still belongs to $\operatorname{Cen}_{G}(L)$. (Note, $L^{\prime} \in \operatorname{Cen}_{G}(L)$ and $L^{\prime} \leqslant_{\mathbf{z} G} L^{\prime \prime} \leqslant \mathbf{z}_{G} L$, then $L^{\prime \prime} \in \operatorname{Cen}_{G}(L)$.) This check (which is different from the original check in [13], [14]) only involves standard operation with integral matrices. The union $\operatorname{Cen}(L)$ resp. $\widehat{\operatorname{Cen}}(L)$ was not formally constructed in step (i), but rather the sequence of the various $\operatorname{Cen}_{G}(L)$ was used as input for step (iii). In some cases of groups with only one dimensional constituent groups $\operatorname{Cen}_{G}(L)$ became so big that there was not enough central memory available to store all the matrices. In these cases we partitioned $\mathrm{Cen}_{G}(L)$ as follows: we chose a set $I$ of nonprimitive orthogonal idempotents of $\mathbf{Q} G$, computed $\operatorname{Cen}_{G}\left(e^{\prime} L\right)=\left\{M \leqslant{ }_{\mathbf{z} G} e^{\prime} L \mid e M=e e^{\prime} L\right.$ for all primitive central idempotents $e$ of $\mathbf{Q} G\}$ for each $e^{\prime} \in I$ by the same program, and partitioned $\operatorname{Cen}_{G}\left(e^{\prime} L\right)$ as $\cup \mathrm{Cen}_{G}(X)$, where

$$
X \in\left\{\bigoplus_{e^{\prime} \in I} X_{e^{\prime}} \mid X_{e^{\prime}} \in \operatorname{Cen}_{G}\left(e^{\prime} L\right)\right\}
$$

and

$$
\operatorname{Cen}_{G}(X)=\left\{M \leqslant_{\mathbf{z} G} X \mid e^{\prime} M=e^{\prime} X \text { for all } e^{\prime} \in I\right\} .
$$

Also, $\operatorname{Cen}_{G}(X)$ was computed by the same program.

Step (iii) has one part which was done by hand, namely to find finitely many generators for the normalizer $N$ of $B$ in $G L_{n}(\mathbf{Z})$, and a machine part, namely to find the orbits on $\operatorname{Cen}(L)$. Though not all of $\operatorname{Cen}(L)$ came as input from step (ii) and some lattices (in the form of matrices describing different bases of the same lattice) more than once, the latter part was routine work. We overcame possible storage shortage by using an important invariant of the $\mathrm{N}$-orbits, which was available from the output of (ii) in the form of elementary divisors of the matrices describing the lattice bases: if $M, M^{\prime} \in \operatorname{Cen}(L)$ lie in the same $N$-orbit, then $L / M$ and $L / M^{\prime}$ are isomorphic abelian groups. As for the generators of $N$, we employed the methods of [2] and [8]. In some cases of infinite normalizers we need not bother to find generators for the full group $N$, since it turned out that the orbits under a proper subgroup of $N$, generators of which were cheaply available, were already distinguished by the invariant just mentioned.

Step (iv) again is routine with the inpul of (iii): Schreier-generators, cf., e.g., [2], for the stabilizer $H(M)$ of the representative lattice $M$ of step (iii) are formed successively and are checked for redundancy. The resulting generators are transformed, thus yielding generators for the desired Bravais group.

III. Examples. The Bravais groups $B$ with natural representation $\Delta: B \rightarrow G L_{n}(\mathbf{Q})$ : $g \mapsto g$ equivalent to $m_{1} \Delta_{1}+m_{2} \Delta_{2}, m_{1}, m_{2} \in \mathbf{N}$ and $\Delta_{1}, \Delta_{2}$ inequivalent irreducible rational representations of $B$ form a good example to demonstrate the procedure of Section II. Clearly an almost decomposable Bravais group with this property is Z-equivalent to

$$
B_{1} \oplus B_{2}:=\left\{\operatorname{diag}\left(g_{1}, g_{2}\right) \mid g_{i} \in B_{i} \text { for } i=1,2\right\},
$$

where $B_{1}$ and $B_{2}$ are suitable Bravais groups of degree $m_{i} \cdot \operatorname{degree}\left(\Delta_{i}\right)$ for $i=1 \mathrm{resp}$. 2. Denote the natural lattice of $B_{i}$ by $L_{i}$ and by $\pi_{i}$ the projection of $L_{1} \oplus L_{2}$ onto $L_{i}$ $(i=1,2)$. By Section II or by [10] clearly every Bravais group $B$ having $B_{1} \oplus B_{2}$ as 
its associated almost decomposable Bravais group (i.e. $B_{1} \oplus B_{2} \in P(B)$ ), arises from a subgroup $L$ of $L_{1} \oplus L_{2}$ with the following properties:

(i) $\left|B_{1}\right| \cdot\left|B_{2}\right| \cdot\left(L_{1} \oplus L_{2}\right) \leqslant L \leqslant L_{1} \oplus L_{2}$;

(ii) $\pi_{1}(L)=L_{1}$ and $\pi_{2}(L)=L_{2}$;

(iii) $\tilde{B}=\left\{g \in B_{1} \oplus B_{2} \mid g L=L\right\}$ has $B_{1} \oplus B_{2}$ as its Bravais group;

(iv) the action of $\tilde{B}$ on $L$ yields the $\mathbf{Z}$-class of Bravais groups containing $B$.

We analyze this in the spirit of [9, Chapter II]: $L$ is a subdirect product of $L_{1}$ and $L_{2}$; namely, with $A:=L_{1} \oplus L_{2} / L$ (called amalgamating factor module) we have $\tilde{B}$-module epimorphisms $\varphi_{i}: L_{i} \rightarrow A$ such that

$$
L=\operatorname{ker} \varphi_{1} \oplus \varphi_{2}=\left\{\left(l_{1}, l_{2}\right) \in L_{1} \oplus L_{2} \mid \varphi_{1}\left(l_{1}\right)=\varphi_{2}\left(l_{2}\right)\right\} .
$$

This subdirect product structure imposes a subdirect product structure on $\tilde{B}$ (or $B$ ): Let $H_{i}=\left\{g \in B_{i} \mid g \operatorname{ker} \varphi_{i}=\operatorname{ker} \varphi_{i}\right\}$ for $i=1,2$. The natural action of $H_{i}$ on $L_{i}$ induces via the embedding of $L_{i}$ into $L_{1} \oplus L_{2}$ and the natural epimorphism of $L_{1} \oplus L_{2}$ onto $A$ an action of $H_{i}$ on $A$ for $i=1,2$. The description of $\tilde{B}$ as subdirect product is given by

$$
\tilde{B}=\left\{\operatorname{diag}\left(g_{1}, g_{2}\right) \mid g_{i} \in H_{i}, g_{1} a=g_{2} a \text { for all } a \in A\right\} .
$$

(We do not claim that all elements of $H_{i}$ turn up as components of elements of $\tilde{B}$, though they usually do in small dimensions.)

This analysis shows how a good knowledge of $B_{1}, B_{2}$, the subgroups of $B_{1}$ and $B_{2}$, and their actions on $L_{1}$ and $L_{2}$ helps to find the Bravais groups $B$ with $B_{1} \oplus B_{2} \in$ $P(B)$; namely one has to watch out for the following situation:

Find subgroups $H_{i}$ of $B_{i}$ for $i=1,2$ satisfying

(i) $B\left(H_{i}\right)=B_{i}, i=1,2$;

(ii) There are $H_{i}$-sublattices $L_{i}^{\prime}$ of finite index in $L_{i}$ with

( $\alpha) L_{1} / L_{1}^{\prime} \cong L_{2} / L_{2}^{\prime}$ as abelian groups,

( $\beta$ ) the actions of $H_{i}$ on $L_{i} / L_{i}^{\prime}$ are compatible in the sense that there is an isomorphism $\varphi: L_{1} / L_{1}^{\prime} \rightarrow L_{2} / L_{2}^{\prime}$ with $\varphi^{-1} \bar{H}_{1} \varphi=\bar{H}_{2}$, where $\bar{H}_{i}$ is the image of $H_{i}$ in $\operatorname{Aut}\left(L_{i} / L_{i}^{\prime}\right)$ induced by the action of $H_{i}$ on $L_{i} / L_{i}^{\prime}$ for $i=1,2$.

( $\gamma$ ) In case both $H_{i}$ act faithfully on $L_{i} / L_{i}^{\prime}$, conjugation by $\varphi$ (defined in $(\beta)$ ) does not transform the irreducible constituent of the $H_{1}$-character afforded by $L_{1}$ onto the irreducible constituent of the $H_{2}$-character afforded by $L_{2}$.

In such a situation, the Bravais group depends only on $L_{1}^{\prime}, L_{2}^{\prime}$ and $\varphi$ and is given as described above. (The other conditions only make sure that $B(\tilde{B})=B_{1} \oplus B_{2}$ in the terminology used above.) It is not necessary to say anything about the action of the normalizer of $B_{1} \oplus B_{2}$, since our point was, how to avoid finding $\operatorname{Min}\left(B_{1} \oplus B_{2}\right)$ in the last chapter.

The above remarks are helpful to understand some of the phenomena reflected in the table in Section IV: If there is just one $\mathbf{Z}$-class of Bravais groups associated with a Z-class of almost decomposable Bravais groups (namely this class itself), then $L_{1}$ and $L_{2}$ have no common epimorphic image compatible with actions of sufficiently big subgroups $H_{i}$ of $B_{i}$ (cf., e.g., families XL, XLI, XLIV, LI etc.). If all Bravais groups associated with some almost decomposable Bravais group $B$ are rationally equivalent to $B$ (and not to a proper subgroup) then the amalgamating factor module $A \cong L_{1} / L_{1}^{\prime} \cong L_{2} / L_{2}^{\prime}$ is centralized by $B_{1}$ and $B_{2}$ (cf., e.g., families II-VI etc.). We leave it as a quick exercise to the reader to prove with the help of the above 
remarks and the normalizer action (step (iv) of Section II) that there are $1+k$ Z-classes of Bravais groups with $\left\langle-I_{n}, \operatorname{diag}\left(-I_{k}, I_{n-k}\right)\right\rangle\left(\cong V_{4}\right)$ as associated almost decomposable Bravais group $1 \leqslant k \leqslant n / 2$. ( $I_{k}$ denotes the $k \times k$-unit matrix.)

We close the section with a curious example at the other extreme of what was discussed above. (In the terminology of Definition (II.1) all $e L \neq 0$ instead of just two of them.)

(III.1) Example. Let $G$ be a finite group, $\rho: G \rightarrow G L_{|G|}(\mathbf{Z})$ the left regular representation of $G$. Assume that $G$ is generated by elements of order 2. Then $B(\rho(G))=\left\langle-I_{|G|}, \rho(G)\right\rangle$. Moreover $\rho(G)$ is Bravais-minimal.

Proof. $\rho(G)$ consists of permutation matrices. Hence $S(\rho(G))$ contains $I_{|G|}$ and $J_{|G|}$, the latter being the $|G| \times|G|$-matrix with all entries equal to 1 . Hence $B(\rho(G))$ consists, up to sign, of permutation matrices. For these the transposed is equal to the inverse. Let $\rho^{\prime}$ denote the right regular representation of $G$. Then the (permutation) matrices in $B(\rho(G))$ centralize $\rho^{\prime}(g)$ for every involution $g \in G$. But $\rho(G)$ is the centralizer of $\rho^{\prime}(G)$ in the group of $|G| \times|G|$-permutation matrices. Hence the first claim follows. The second statement follows since $\left.\rho\right|_{H}$ has more $\mathbf{Q}$-irreducible constituents (multiplicities counted) as $\rho$ has for every proper subgroup $H$ of $G$. Hence $B(\rho(H)) \supsetneqq B(\rho(G))$, cf., e.g., [9]. Q.E.D.

IV. A Symbol for Crystal Families, Results in Six Dimensions. The list at the end of this section gives a survey of the six-dimensional lattices resp. Bravais groups as they are distributed into crystal families. The latter are the equivalence classes of the join of the following two equivalence relations on the set of finite unimodular groups: Q-equivalence and "having the same Bravais group", cf., e.g., [6]. As already derivable from [4] and [10] there are altogether 91 crystal famililes in six dimensions. It is useful to have informative names or symbols for crystal families. We describe how such a symbol can be put together from "atomic symbols". To this end we have to recall the definition of the generalized Bravais groups $B_{I}(G)$ for finite subgroups $G$ of $G L_{n}(\mathbf{Z})$ from [8]:

and

$$
V_{I}(G)=\left\{X \in \mathbf{R}^{n \times n} \mid g^{t r} X g=X \text { for all } g \in G\right\}
$$

$$
B_{I}(G)=\left\{g \in G L_{n}(\mathbf{Z}) \mid g^{t r} X g=X \text { for all } X \in V_{I}(G)\right\} .
$$

The generalized Bravais groups $G\left(=B_{I}(G)\right)$ give rise to the definition of the strict crystal families, which we define in exactly the same way as crystal families a few lines above with the words "Bravais group" replaced by "generalized Bravais group". The symbol for the crystal family is a slight generalization of the decomposition scheme of a crystal family used in [10]: Let $\Delta: G \rightarrow G L_{n}(\mathbf{Q})$ be the natural representation of some group $G$ in a crystal family $\mathfrak{F}$ and assume $\Delta$ splits into pairwise rationally inequivalent, $\mathbf{Q}$-irreducible representations $\Delta_{i}$ of $G$ with positive multiplicities $m_{i}$, i.e., $\Delta \sim \mathbf{Q}+{ }_{i=1}^{s} m_{i} \Delta_{i}$. Order the $\Delta_{i}$ in such a way that the degrees $n_{i}$ of $\Delta_{i}$ satisfy $n_{1} \geqslant \cdots \geqslant n_{s}$ with $n_{i}=n_{i+1}$ implying $m_{i} \geqslant m_{i+1}$. The decomposition scheme of $G$ and-since it is a family invariant-of $\mathfrak{F}$ was defined by the $\left(m_{1}+\cdots+m_{s}\right)$-tuple

$$
\left(\overline{\frac{n_{1}, \ldots, n_{1}}{m_{1}}}, \overline{\frac{n_{2}, \ldots, n_{2}}{m_{2}}}, \ldots, \overline{\frac{n_{s}, \ldots, n_{s}}{m_{s}}}\right)
$$


with the bar over $n_{i}$ omitted in case $m_{i}=1$. To define a family symbol we modify the above tuple by replacing $n_{i}$ by a symbol of the crystal family of $\Delta_{i}(G)$ in case $m_{i}=1$ and by a symbol for the generalized crystal family in case $m_{i}>1$. Of course we have to assume that the $\Delta_{i}$ defined above are in addition integral, which is certainly possible. To make the symbol unique, one has to put some ordering on those strict and ordinary crystal families of each degree which consist of irreducible groups. That the family symbol is well defined and determines its family uniquely, once the "atomic symbols" for the irreducible strict and ordinary families are defined, follows easily from [8].

For these atomic symbols we have adopted the convention of writing the degree with an index - if necessary. Hence 1 and 3 are the symbols of the (unique) irreducible 1- resp. 3-dimensional crystal families. Since these families coincide with the strict families in these dimensions no new names are necessary for the strict families. In dimension 2, we have two irreducible families which we denote by $2_{1}$ and $2_{2}$, the first containing a dihedral group of order 8 as Bravais group (square lattices), the second a dihedral group of order 12 (hexagonal lattices). (Note, now the symbols of all 3-dimensional lattices are quickly derived: $(\overline{1,1,1}),(\overline{1,1}, 1),(1,1,1),(2,1)$, $\left(2_{2}, 1\right)$, (3).) Each of the families $2_{1}$ and $2_{2}$ splits into two strict families which are given the symbol $2_{1}, 2_{1^{\prime}}$ and $2_{2}, 2_{2^{\prime}}$ respectively. The groups in $2_{1^{\prime}}$ and $2_{2^{\prime}}$ are all cyclic, namely of order 4 in the first and of orders 3 and 6 in the second case. No confusion between the strict family $2_{i}$ and the ordinary family $2_{i}$ can arise, since the first (atomic) symbol only occurs underneath a bar in the family symbol, and the second, never. For the symbols of the six-dimensional families we do not need the symbols for the strict irreducible families in dimensions 4,5 , and 6 , but only for the ordinary ones. In dimension 4 we picked $4_{1}, 4_{2}, 4_{3}$ for the three families containing absolutely irreducible groups, namely groups isomorphic to the wreath product $C_{2} \backslash S_{4}$ in the first, $D_{12} \backslash S_{2}$ in the second and $C_{2} \times S_{5}$ in the third case. $4_{1^{\prime}}, 4_{2^{\prime}}$ and $4_{3}$, are the atomic symbols for the families containing $\mathbf{Q}$ - but not $\mathbf{C}$-irreducible groups; family $4_{1}$, contains a dihedral group of order 16 , family $4_{2}$, one of order 24 , and $4_{3}$, one of order 20 . Finally $5_{1}$ and $5_{2}$ are the symbols for the irreducible 5-dimensional families: $5_{1}$ contains a $C_{2} \backslash S_{5}$ and $5_{2}$ a $C_{2} \times S_{6}$. The symbols for the irreducible 6-dimensional families can be read from the table.

We now have a convenient terminology to explain how to find the almost decomposable Bravais groups, as promised in Section II. With the atomic symbols for the irreducible crystal families in dimensions smaller or equal to 6 , and for the strict irreducible families in dimensions less than 4 , it is a combinatorial task to enumerate the symbols for the 6-dimensional families. The almost decomposable Bravais groups in a family whose symbol does not have a bar are completely decomposable and direct products of irreducible Bravais groups $B_{i}$, namely $B_{1} \oplus$ $\cdots \oplus B_{s}$ in the terminology of Section III. It is clear that the Bravais groups in families whose symbol has only bars above the 1's, are completely decomposable. For the other families, we have to employ the methods and results of [9] to see that the only 6-dimensional families with almost decomposable Bravais groups which are not completely decomposable are the ones with symbol $(\overline{3,3})$ or involving $\overline{2_{1}, 2_{1}}$ as a partial symbol. By means of [9] one can also write down the Z-classes of almost decomposable Bravais groups in these cases. 
In the following table of six-dimensional Bravais groups which can also be used as a guide to the microfiche included in this issue, the family symbol explained at the beginning of this chapter is given in the second column. This symbol does not appear on the microfiche, but only the family number of column one. Column 3 lists the dimensions of the spaces $S(G)$ of quadratic forms fixed by the groups $G$ in the family. The number of $\mathbf{Z}$-classes and the isomorphism types of the almost decomposable Bravais groups in the family are given in columns 4 and 5 . The conventions for the isomorphism types are as follows: exponents give the number of isomorphic direct factors, $C_{n}, D_{2 n}, S_{n}, A_{n}$ denote cyclic, dihedral, symmetric or alternating groups of order $n, 2 n$ resp. of degree $n$, । denotes wreath products, $W\left(D_{n}\right), W\left(E_{6}\right)$, $W\left(F_{4}\right)$ denote the Weyl groups of the root systems $D_{n}, E_{6}$ and $F_{4}$. The last column gives the number of Z-classes of Bravais groups of the family in the form $r_{1}\left(r_{1}^{\prime}\right)+$ $\cdots+r_{s}\left(r_{s}^{\prime}\right)$, where the $r_{i}$ is the number of $\mathbf{Z}$-classes of Bravais groups associated with the $i$ th $\mathbf{Z}$-class of almost decomposable Bravais groups in the family (in some order), and $r_{i}^{\prime}$ counts those $\mathbf{Z}$-classes among these, the groups of which are Q-equivalent to a proper subgroup of the associated almost decomposable Bravais group; $\left(r_{i}^{\prime}\right)$ is omitted if $r_{i}^{\prime}=0$.

On the attached microfiche, representatives $B$ of the $\mathbf{Z}$-classes of Bravais groups are given by generating matrices, their orders, and $\mathbf{R}$-bases of the corresponding space $S(B)$ of quadratic forms. They are ordered by families, inside a family according to associated almost decomposable Bravais groups. Bases for the representative lattices in $\operatorname{Cen}(L)$ (cf. Section II) which define the Bravais groups via the associated almost decomposable Bravais groups are given in the form of matrices, together with the inverses and elementary divisors of these matrices (the latter being a helpful invariant for recognition). In case the Bravais group $B$ is Q-equivalent to a proper subgroup $\tilde{B}$ of its associated almost decomposable Bravais group, generators of $\tilde{B}$ are also given.

\section{6-DIMENSIONAL BRAVAIS GROUPS}

\begin{tabular}{|c|c|c|c|c|c|}
\hline $\begin{array}{l}\text { family } \\
\text { number }\end{array}$ & $\begin{array}{l}\text { family } \\
\text { symbol }\end{array}$ & $\begin{array}{l}\text { number } \\
\text { of para- } \\
\text { meters }\end{array}$ & $\begin{array}{l}\text { numb. of } \\
Z-c l . \text { of } \\
\text { a.d.Br.gr. }\end{array}$ & $\begin{array}{l}\text { isom.types of } \\
\text { a.d.Bravais gr. }\end{array}$ & $\begin{array}{l}\text { number of } \\
\mathbf{Z}-c l \text { asses } \\
\text { of Bravais } \\
\text { groups }\end{array}$ \\
\hline I & $(\overline{1,1,1,1,1,1})$ & 21 & 1 & $c_{2}$ & 1 \\
\hline I I & $(\overline{1,1,1,1,1}, 1)$ & 16 & 1 & $\mathrm{c}_{2}{ }^{2}$ & 2 \\
\hline I I I & $(\overline{1,1,1,1}, \overline{1,1})$ & 13 & 1 & $\mathrm{C}_{2}{ }^{2}$ & 3 \\
\hline IV & $(\overline{1,1,1}, \overline{1,1,1})$ & 12 & 1 & $\mathrm{C}_{2}{ }^{2}$ & 4 \\
\hline $\mathrm{v}$ & $\langle\overline{1,1,1,1}, 1,1\rangle$ & 12 & 1 & $\mathrm{c}_{2}^{3}$ & 6 \\
\hline VI & $(2, \overline{1,1,1,1})$ & 11 & 1 & $\mathrm{D}_{8} \times \mathrm{C}_{2}$ & 2 \\
\hline VII & $(2, \overline{1,1,1,1})$ & 11 & 1 & $\mathrm{D}_{12} \times \mathrm{C}_{2}$ & $2(1)$ \\
\hline VIII & $(\overline{1,1,1}, \overline{1,1}, 1)$ & 10 & 1 & $\mathrm{C}_{2}^{3}$ & 12 \\
\hline IX & $(\overline{1,1}, \overline{1,1}, \overline{1,1})$ & 9 & 1 & $\mathrm{C}_{2}^{3}$ & 12 \\
\hline $\mathrm{x}$ & $(\overline{1,1,1}, 1,1,1)$ & 9 & 1 & $c_{2}^{4}$ & $19(1)$ \\
\hline$X I$ & $\left(\overline{2_{1},, 2_{1}, 2_{1}}\right)$ & 9 & 1 & $\mathrm{C}_{4}$ & 1 \\
\hline $\mathrm{XII}$ & $\left(\overline{2} z^{\prime},{ }^{2} z,{ }^{2} z\right)$ & 9 & 1 & $c_{6}$ & 1 \\
\hline
\end{tabular}




\section{6-DIMENSIONAL BRAVAIS GROUPS}

\begin{tabular}{|c|c|c|c|c|c|}
\hline $\begin{array}{l}\text { family } \\
\text { number }\end{array}$ & $\begin{array}{l}\text { family } \\
\text { symbol }\end{array}$ & $\begin{array}{l}\text { number } \\
\text { of para- } \\
\text { meters }\end{array}$ & $\begin{array}{l}\text { numb. of } \\
Z-c l . \text { of } \\
a \cdot d \cdot B r \cdot g r .\end{array}$ & $\begin{array}{l}\text { isom.types of } \\
\text { a.d.Bravais gr. }\end{array}$ & $\begin{array}{l}\text { number of } \\
\mathbf{Z} \text {-classes } \\
\text { of Bravais } \\
\text { groups }\end{array}$ \\
\hline$X I I I$ & $(\overline{1,1}, \overline{1,1}, 1,1)$ & 8 & 1 & $\mathrm{C}_{2}{ }^{4}$ & $39(2)$ \\
\hline$X I V$ & $(2, \overline{1,1,1}, 1)$ & 8 & 1 & $\mathrm{D}_{8} \times \mathrm{C}_{2}{ }^{2}$ & $8(1)$ \\
\hline$X V$ & $(2, \overline{1,1,1}, 1)$ & 8 & 1 & $\mathrm{D}_{12} \times \mathrm{C}_{2}{ }^{2}$ & $6(4)$ \\
\hline XVI & $(\overline{1,1}, 1,1,1,1)$ & 7 & 1 & $c_{2}^{5}$ & $57(8)$ \\
\hline XVII & $(2, \overline{1,1}, \overline{1,1})$ & 7 & 1 & $\mathrm{D}_{8} \times \mathrm{C}_{2}{ }^{2}$ & $11(2)$ \\
\hline XVIII & $(2, \overline{1,1}, \overline{1,1})$ & 7 & 1 & $\mathrm{D}_{12} \times \mathrm{C}_{2}{ }^{2}$ & $6(3)$ \\
\hline $\mathrm{XIX}$ & $\left(\overline{2_{1}, 2_{1}}, \overline{1,1}\right)$ & 7 & 1 & $C_{4} \times C_{2}$ & 3 \\
\hline $\mathrm{xx}$ & $\left(\overline{2} z^{\prime 2} 2^{\prime}, \overline{1,1}\right)$ & 7 & 1 & $c_{6} \times C_{2}$ & $3(2)$ \\
\hline$X X I$ & $(3, \overline{1,1,1})$ & 7 & 3 & $\left(c_{2} \sim S_{3}\right) \times C_{2}$ & $3(1)+2+1$ \\
\hline XXII & $(1,1,1,1,1,1)$ & 6 & 1 & $c_{2}{ }^{6}$ & $49(13)$ \\
\hline$X X I I I$ & $(2, \overline{1,1}, 1,1)$ & 6 & 1 & $\mathrm{D}_{8} \times \mathrm{C}_{2}{ }^{3}$ & $35(8)$ \\
\hline $\mathrm{XXIV}$ & $(2,1,1,1,1)$ & 6 & 1 & $\mathrm{D}_{12} \times \mathrm{C}_{2}{ }^{3}$ & $19(13)$ \\
\hline$x x V$ & $\left(\overline{2_{1},, 2_{1}}, 1,1\right)$ & 6 & 1 & $\mathrm{C}_{4} \times \mathrm{C}_{2}{ }^{2}$ & $7(1)$ \\
\hline XXVI & $\left(\overline{2_{2}, 2_{2}}, 1,1\right)$ & 6 & 1 & $c_{6} \times c_{2}^{2}$ & $4(2)$ \\
\hline XXVII & $(\overline{2}, 2, \overline{1}, \overline{1})$ & 6 & 3 & $\mathrm{D}_{8} \times \mathrm{C}_{2}$ & $3+4+2$ \\
\hline XXVIII & $\left(\overline{2_{2},{ }_{2}}, \overline{1,1}\right)$ & 6 & 2 & $\mathrm{D}_{12} \times \mathrm{C}_{2}$ & $3(2)+2(1)$ \\
\hline$X X I X$ & $\left(\overline{2_{1}, 2_{1}, 2_{1}}\right)$ & 6 & 3 & $\mathrm{D}_{8}$ & $1+1+1$ \\
\hline $\mathrm{XXX}$ & $\left(\overline{2_{2},{ }^{2},^{2}{ }_{2}}\right)$ & 6 & 2 & $\mathrm{D}_{12}$ & $1+1$ \\
\hline $\mathrm{XXXI}$ & $(2,1,1,1,1)$ & 5 & 1 & $\mathrm{D}_{8} \times \mathrm{C}_{2}^{4}$ & $46(16)$ \\
\hline$X X X I I$ & $(2,1,1,1,1)$ & 5 & 1 & $\mathrm{D}_{12} \times \mathrm{C}_{2}^{4}$ & $22(14)$ \\
\hline $\mathrm{XXX} I I I$ & $\left(\overline{2_{1}, 2_{1}}, 1,1\right)$ & 5 & 3 & $\mathrm{D}_{8} \times \mathrm{C}_{2}{ }^{2}$ & $8(2)+11(1)+7(2)$ \\
\hline$X X X I V$ & $\left(\overline{2}{ }^{2},{ }_{2}, 1,1\right)$ & 5 & 2 & $\mathrm{D}_{12} \times \mathrm{C}_{2}{ }^{2}$ & $4(2)+6(4)$ \\
\hline $\mathrm{XXXV}$ & $(2,, 2, \overline{1,1})$ & 5 & 1 & $\mathrm{D}_{8}^{2} \times \mathrm{C}_{2}$ & $10(4)$ \\
\hline$X X X V I$ & $(2,2, \overline{1,1})$ & 5 & 1 & $\mathrm{D}_{12}{ }^{2} \times \mathrm{C}_{2}$ & $8(7)$ \\
\hline XXXVII & $(2,2, \overline{2}, \overline{1,1})$ & 5 & 1 & $\mathrm{D}_{8} \times \mathrm{D}_{12} \times \mathrm{C}_{2}$ & $4(2)$ \\
\hline XXXVIII & $\left(\overline{2_{1},, 2_{1}}, 2_{1}\right)$ & 5 & 1 & $C_{4} \times D_{8}$ & $3(1)$ \\
\hline $\operatorname{XXXIX}$ & $\left(\overline{2_{2}, 2_{2}}, 2_{2}\right)$ & 5 & 1 & $C_{6} \times D_{12}$ & $3(2)$ \\
\hline$X L$ & $\left(\overline{2_{1},, 2_{1}}, 2_{2}\right)$ & 5 & 1 & $\mathrm{C}_{4} \times \mathrm{D}_{12}$ & 1 \\
\hline$X L I$ & $\left(\overline{2_{2},, 2_{2}}, 2_{1}\right)$ & 5 & 1 & $\mathrm{C}_{6} \times \mathrm{D}_{8}$ & 1 \\
\hline XLII & $(3, \sqrt[1,1]{1}, 1)$ & 5 & 3 & $\left(c_{2} \sim s_{3}\right) \times C_{2}{ }^{2}$ & $11(4)+7+2$ \\
\hline$X L I I I$ & $(4,1, \overline{1,1})$ & 5 & 1 & $D_{16} \times C_{2}$ & 2 \\
\hline XLIV & $\left(4_{2}, \overline{1,1}\right)$ & 5 & 1 & $\mathrm{D}_{24} \times \mathrm{C}_{2}$ & 1 \\
\hline XLV & $\left(4_{3},, \overline{1,1}\right)$ & 5 & 1 & $\mathrm{D}_{20} \times \mathrm{C}_{2}$ & $2(1)$ \\
\hline XLVI & $(2,2,2,1,1)$ & 4 & 1 & $\mathrm{D}_{8}{ }^{2} \times \mathrm{C}_{2}{ }^{2}$ & $32(17)$ \\
\hline XLVII & $(2,2,1,1)$ & 4 & 1 & $\mathrm{D}_{12}{ }^{2} \times \mathrm{C}_{2}{ }^{2}$ & $18(16)$ \\
\hline
\end{tabular}




\section{6-DIMENSIONAL BRAVAIS GROUPS}

\begin{tabular}{|c|c|c|c|c|c|}
\hline $\begin{array}{l}\text { family } \\
\text { number }\end{array}$ & $\begin{array}{l}\text { family } \\
\text { symbol }\end{array}$ & $\begin{array}{l}\text { number } \\
\text { of para- } \\
\text { meters }\end{array}$ & $\begin{array}{l}\text { numb. of } \\
Z-c l \cdot \text { of } \\
a \cdot d \cdot B r \cdot g r .\end{array}$ & $\begin{array}{l}\text { isom.types of } \\
\text { a.d.Bravais gr. }\end{array}$ & $\begin{array}{l}\text { number of } \\
Z \text {-classes } \\
\text { of Bravais } \\
\text { groups }\end{array}$ \\
\hline XLVIII & $(2,2,2,1,1)$ & 4 & 1 & $\mathrm{D}_{8} \times \mathrm{D}_{12} \times \mathrm{C}_{2}{ }^{2}$ & $13(8)$ \\
\hline IL & $\left(\overline{2_{1}, 2_{1}}, 2_{1}\right)$ & 4 & 3 & $\mathrm{D}_{8}{ }^{2}$ & $4(2)+4(1)+4(2)$ \\
\hline L & $\left(\overline{2_{2},{ }^{2}}, 2_{2}\right)$ & 4 & 2 & $\mathrm{D}_{12}{ }^{2}$ & $3(2)+3(2)$ \\
\hline LI & $\left(\overline{2_{1}, 2_{1}}, 2_{2}\right)$ & 4 & 3 & $D_{8} \times D_{12}$ & $1+1+1$ \\
\hline LII & $\left(\overline{2_{2}, 2_{2}}, 2_{1}\right)$ & 4 & 2 & $D_{12} \times D_{8}$ & $1+1$ \\
\hline LIII & $(3,1,1,1)$ & 4 & 3 & $\left(c_{2} \sim s_{3}\right) \times c_{2}^{3}$ & $23(11)+12+4$ \\
\hline LIV & $(4,1,1,1)$ & 4 & 1 & $\mathrm{D}_{16} \times \mathrm{C}_{2}{ }^{2}$ & $6(1)$ \\
\hline LV & $\left(4_{2}, 1,1\right)$ & 4 & 1 & $\mathrm{D}_{24} \times \mathrm{C}_{2}{ }^{2}$ & 2 \\
\hline LVI & $\left(4_{3}, 1,1\right)$ & 4 & 1 & $\mathrm{D}_{20} \times \mathrm{C}_{2}{ }^{2}$ & $4(2)$ \\
\hline LVII & $(4, \overline{1,1})$ & 4 & 2 & $\left(C_{2} \sim S_{4}\right) \times C_{2}$ & $2+3(2)$ \\
\hline LVI II & $(4, \overline{1,1})$ & 4 & 2 & $\begin{array}{l}W\left(F_{4}\right) \times C_{2} \\
\left(D_{12} \sim S_{2}\right) \times C_{2}, \\
\left(C_{2} \times\left(D_{6} \sim S_{2}\right)\right) \times C_{2}\end{array}$ & $2(1)+2(1)$ \\
\hline LIX & $\left(4_{3}, \overline{1,1}\right)$ & 4 & 2 & $\left(C_{2} \times S_{5}\right) \times C_{2}$ & $2(1)+1$ \\
\hline LX & $\left(2_{1}, 2_{1}, 2_{1}\right)$ & 3 & 1 & $\mathrm{D}_{8}^{3}$ & $11(7)$ \\
\hline LXI & $\left(2_{2}, 2_{2}, 2_{2}\right)$ & 3 & 1 & & $9(8)$ \\
\hline LXII & $\left(2_{1}, 2_{1}, 2_{2}\right)$ & 3 & 1 & & $3(1)$ \\
\hline LXIII & $\left(2_{1}, 2_{2}, 2_{2}\right)$ & 3 & 1 & $D_{8} \times D_{12}$ & $3(2)$ \\
\hline LXIV & $(3,2,1,1)$ & 3 & 3 & $\left(C_{2} \sim S_{3}\right) \times D_{8} \times C_{2}$ & $10(4)+6+2$ \\
\hline LXV & $(3,2,1)$ & 3 & 3 & $\left(C_{2} i S_{3}\right) \times D_{12} \times C_{2}$ & $3(1)+4(2)+2(1)$ \\
\hline LXVI & $(\overline{3,3})$ & 3 & 9 & $\mathrm{C}_{2} \sim \mathrm{S}_{3}$ & $9=1+\ldots+1$ \\
\hline LXVII & $\left(4_{1},, 2_{1}\right)$ & 3 & 1 & $D_{16} \times D_{8}$ & $3(1)$ \\
\hline LXVIII & $\left(4_{2}, 2_{1}\right)$ & 3 & 1 & $D_{24} \times D_{8}$ & $2(1)$ \\
\hline $\operatorname{LXIX}$ & $\left(4_{3}, 2_{1}\right)$ & 3 & 1 & $\mathrm{D}_{20} \times \mathrm{D}_{8}$ & 1 \\
\hline LXX & $\left(4_{1}, 2_{2}\right)$ & 3 & 1 & $\mathrm{D}_{16} \times \mathrm{D}_{12}$ & 1 \\
\hline LXXI & $\left(4_{2}, 2_{2}\right)$ & 3 & 1 & $\mathrm{D}_{24} \times \mathrm{D}_{12}$ & $2(1)$ \\
\hline LXXII & $\left(4_{3},, 2_{2}\right)$ & 3 & 1 & $\mathrm{D}_{20} \times \mathrm{D}_{12}$ & 1 \\
\hline LXXI I I & $(4,1,1)$ & 3 & 2 & $\begin{array}{l}\left(\mathrm{C}_{2} \sim \mathrm{S}_{4}\right) \times \mathrm{C}_{2}{ }^{2}, \\
\mathrm{~W}\left(\mathrm{~F}_{4}\right) \times \mathrm{C}_{2}{ }^{2}\end{array}$ & $6(1)+6(4)$ \\
\hline LXXIV & $(4,1,1)$ & 3 & 2 & 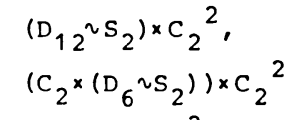 & $6(4)+4(2)$ \\
\hline LXXV & $(4,1,1)$ & 3 & 2 & $\left(C_{2} \times S_{5}\right) \times C_{2}^{2}$ & $6(4)+2$ \\
\hline LXXVI & $\left(6_{2},\right)$ & 3 & 1 & $\mathrm{D}_{36}$ & 1 \\
\hline LXXVII & $\left(6_{3},\right)$ & 3 & 1 & $\mathrm{D}_{28}$ & 1 \\
\hline
\end{tabular}




\begin{tabular}{|c|c|c|c|c|c|}
\hline $\begin{array}{l}\text { family } \\
\text { number }\end{array}$ & $\begin{array}{l}\text { family } \\
\text { symbol }\end{array}$ & $\begin{array}{l}\text { number } \\
\text { of para- } \\
\text { meters }\end{array}$ & $\begin{array}{l}\text { numb. of } \\
\text { z-cl. of } \\
\text { a.d.Br.gr. }\end{array}$ & $\begin{array}{l}\text { isom.types of } \\
\text { a.d.Bravais gr. }\end{array}$ & $\begin{array}{l}\text { number of } \\
\text { Z-classes } \\
\text { of Bravais } \\
\text { groups }\end{array}$ \\
\hline LXXVI I I & $(3,3)$ & 2 & 6 & $\left(c_{2} \sim s_{3}\right)^{2}$ & $\begin{array}{r}4(2)+2+1+5(3)+ \\
2(1)+5(4)\end{array}$ \\
\hline LXXIX & $\left(4,2,{ }_{1}\right)$ & 2 & 2 & $\begin{array}{l}\left(\mathrm{C}_{2} \sim \mathrm{S}_{4}\right) \times \mathrm{D}_{8} \\
\mathrm{~W}\left(\mathrm{~F}_{4}\right) \times \mathrm{D}_{8}\end{array}$ & $3(1)+3(2)$ \\
\hline LXXX & $\left(4,2,{ }_{1}\right)$ & 2 & 2 & $\begin{array}{l}\left(D_{12}{ }^{\sim S_{2}}\right) \times D_{8}, \\
\left(C_{2} \times\left(D_{6} \sim S_{2}\right)\right) \times D_{8}\end{array}$ & $3(2)+1$ \\
\hline LXXXI & $\left(4_{3}, 2_{1}\right)$ & 2 & 2 & $\left(C_{2} \times S_{5}\right) \times D_{8}$ & $1+2(1)$ \\
\hline LXXXII & $\left(4_{1}, 2_{2}\right)$ & 2 & 2 & $\begin{array}{l}\left(\mathrm{C}_{2} \sim \mathrm{S}_{4}\right) \times \mathrm{D}_{12}, \\
\mathrm{~W}\left(\mathrm{~F}_{4}\right) \times \mathrm{D}_{12}\end{array}$ & $1+1$ \\
\hline LXXXIII & $\left.\left(4_{2},{ }^{2}\right)^{\prime}\right)$ & 2 & 2 & $\begin{array}{l}\left(D_{12} \sim S_{2}\right) \times D_{12} \prime \\
\left(C_{2} \times\left(D_{6} \sim S_{2}\right)\right) \times D_{12}\end{array}$ & $3(2)+3(2)$ \\
\hline LXXXIV & $\left(4_{3},{ }_{2}\right)$ & 2 & 2 & $\left(C_{2} \times S_{5}\right) \times D_{12}$ & $1+1$ \\
\hline $\operatorname{LxxxV}$ & $(5,1)$ & 2 & 3 & $\left(C_{2} \sim S_{5}\right) \times C_{2}$ & $2+1+3(1)$ \\
\hline LXXXVI & $(5,, 1)$ & 2 & 4 & $\left(C_{2} \times S_{6}\right) \times C_{2}$ & $4(2)+1+2(1)+2$ \\
\hline LXXXVII & $\left(6_{4},\right)$ & 2 & 3 & $C_{2} \times A_{5}$ & $1+1+1$ \\
\hline LXXXVIII & $\left(6_{1}\right)$ & 1 & 6 & $\begin{array}{l}\mathrm{C}_{2} \sim \mathrm{S}_{6^{\prime}} \\
\left(\mathrm{C}_{2} \sim \mathrm{S}_{3}^{\prime}\right) \sim \mathrm{S}_{2^{\prime}} w\left(\mathrm{D}_{6}\right)\end{array}$ & $6=1+\ldots+1$ \\
\hline LXXXIX & $\left(6_{2}\right)$ & 1 & 5 & $\begin{array}{l}D_{12} \sim S_{3}, C_{2} \times W\left(E_{6}\right), \\
D_{12} \times S_{4}\end{array}$ & $5=1+\ldots+1$ \\
\hline $\mathrm{xc}$ & $\left(6_{3}\right)$ & 1 & 3 & $\mathrm{C}_{2} \times \mathrm{S}_{7}, \mathrm{C}_{2} \times \mathrm{PGL}(2,7)$ & $3=1+1+1$ \\
\hline $\mathrm{XCI}$ & $\left(6_{4}\right)$ & 1 & 3 & $\mathrm{C}_{2} \times \mathrm{S}_{5}$ & $3=1+1+1$ \\
\hline
\end{tabular}

V. Concluding Remarks. One of the most interesting questions in our context is, how much the orders of the Bravais groups within a crystal family differ, or, slightly more restricted, how much symmetry one might lose if one passes from the natural lattice $L$ of an almost decomposable Bravais group $B$ to a sublattice (in $\operatorname{Cen}(L)$ ) belonging to a Bravais group associated with $B$. Certainly, the example at the end of Section III shows that the difference might become tremendously big if one is willing to go to sufficiently big dimensions. The biggest index we found in dimension 6 is 48 in families $(3,3)$ and $\left(4_{3}, 2_{1}\right)$. In checking these tables we found a mistake in $[9$, Theorem V.3(ii)]. The subgroup of $G L_{6}(\mathbf{Z})$ given there is not a maximal finite subgroup of $G L_{6}(\mathbf{Z})$, not even a Bravais group as one might check from the attached microfiche under family $(3,3)$. In fact family $(3,3)$ does not seem to contain a maximal finite subgroup of $G L_{6}(\mathbf{Z})$, the constituent groups of which are not maximal finite in $G L_{3}(\mathbf{Z})$. However, the phenomenon to be demonstrated in Theorem V.3(ii) of [9] certainly exists: There are maximal finite reducible subgroups of $G L_{n}(\mathbf{Z})$, the $k$-dimensional constituent groups of which are not maximal finite in $G L_{k}(\mathbf{Z})$ for some $k$. 
(V.1) Example. In the 7-dimensional crystal family with symbol $\left(4_{2}, 3\right)$ there are maximal finite subgroups of $G L_{7}(\mathbf{Z})$, the 4-dimensional constituent groups are not maximal finite in $G L_{4}(\mathbf{Z})$.

Proof. Let $B_{1}$ be the Bravais group with

$$
S\left(B_{1}\right)=\left\langle\left(\begin{array}{ll}
1 & 0 \\
0 & 1
\end{array}\right) \otimes\left(\begin{array}{rr}
2 & -1 \\
-1 & 2
\end{array}\right)\right\rangle
$$

and $B_{2}$ the Bravais group with

$$
S\left(B_{2}\right)=\left\langle\left(\begin{array}{lll}
1 & 0 & 0 \\
0 & 1 & 0 \\
0 & 0 & 1
\end{array}\right)\right\rangle_{\mathbf{R}} \quad \text { or } S\left(B_{2}\right)=\left\langle\left(\begin{array}{lll}
2 & 1 & 1 \\
1 & 2 & 1 \\
1 & 1 & 2
\end{array}\right)\right\rangle_{\mathbf{R}} .
$$

Denote the natural lattices by $L_{1}$ and $L_{2}$. Our maximal finite subgroup will be a Bravais group $B$ with $B_{1} \oplus B_{2}$ as associated almost decomposable Bravais group. The $B_{2}$-lattice $L_{2}$ has a factor module $M$, which is a 2 -dimensional $\mathbf{Z} / 2 \mathbf{Z}$-vector space. $B_{1}$ has a subgroup $H_{1}$ with $B\left(H_{1}\right)=B_{1}$, namely

$$
H_{1}=\left\langle\left(\begin{array}{rr}
-1 & 0 \\
0 & 1
\end{array}\right) \otimes\left(\begin{array}{ll}
0 & -1 \\
1 & -1
\end{array}\right),\left(\begin{array}{ll}
1 & 0 \\
0 & 1
\end{array}\right) \otimes\left(\begin{array}{ll}
0 & 1 \\
1 & 0
\end{array}\right),\left(\begin{array}{ll}
0 & 1 \\
1 & 0
\end{array}\right) \otimes\left(\begin{array}{ll}
1 & 0 \\
0 & 1
\end{array}\right)\right\rangle,
$$

such that $L_{1}$ viewed as an $H_{1}$-lattice has a factor module $N$ isomorphic to $M$ as abelian group. Both, $H_{1}$ and $B_{1}$, induce the full $G L_{2}(\mathbf{Z} / 2 \mathrm{Z})$-action on $N$ resp. $M$. Taking the subdirect product $L$ of $L_{1}$ and $L_{2}$ with $M$ and $N$ identified (as amalgamating factor module) and taking the corresponding subdirect product of $H_{1}$ and $B_{2}$, as described in Section II, yields a Bravais group $B$ with $H_{1}$ as constituent group. Clearly $H_{1}$ is not maximal finite in $G L_{4}(\mathrm{Z})$, since it is of index 6 in $B_{1}$. However, $B$ is maximal finite in $G L_{7}(Z)$. Namely, one only has to check that $B$ is not contained in an irreducible maximal finite subgroup of $G L_{7}(\mathbf{Z})$. The spaces of forms for these groups are generated by integral primitive matrices, the elementary divisors only involve powers of 2 , cf. [13]. But in $S(B)$ there are no nonsingular integral matrices with this property, since there is always a 3 involved in the elementary divisors (coming from $\left.\left(\begin{array}{ll}1 & 0 \\ 0 & 1\end{array}\right) \otimes\left(\begin{array}{cc}2 & -1 \\ -1 & 2\end{array}\right)\right)$. Q.E.D.

Our tables do not give immediate insight about inclusions of Bravais groups and therefore one might wish more information of this kind to gain some insight into the geometry of the space of quadratic forms in six dimensions. How a combinatorial aspect of the geometric picture might be approached is the last topic of this paper.

Let $S^{\prime}(G)$ denote the subset of all positive definite matrices in $S(G)$ for any finite unimodular group $G$ and call the sets $S$ of the form $S=S^{\prime}(G)$ Bravais manifolds. The intersection of two Bravais manifolds is either empty or again a Bravais manifold. Hence the set $\mathfrak{B}_{n}$ of all Bravais manifolds of finite subgroups of $G L_{n}(\mathbf{Z})$ together with the empty set is a semigroup with respect to intersection. The conjugation action of $G L_{n}(\mathbf{Z})$ on the set $\mathfrak{B}_{n}^{\prime}$ of Bravais groups of degree $n$ is similar to the action on $\mathfrak{B}_{n} \backslash\{\phi\}$ via $S \mapsto g^{t r} S g$ for $g \in G L_{n}(\mathbf{Z})$ and $S \in \mathfrak{B}_{n} \backslash\{\phi\}$. For the sake of clarity, we describe the construction we are going to suggest first in an abstract context.

(V.2) Remark. Let $M$, ○ be a semigroup and $G$ a group acting as an automorphism group on $M$. Assume

(i) For each $m \in M$ there are only finitely many $x, y \in M$ with $x \circ y=m$; 
(ii) $M / G$ is finite, i.e., $G$ has only finitely many orbits on $M$.

Then there is a ring with $\mathbf{Z}$-basis $\left(b_{O} \mid O \in M / G\right)$ of cardinality $|M / G|$ such that

$$
b_{O_{1}} b_{O_{2}}=\sum_{O_{3} \in M / G} \alpha\left(O_{1}, O_{2}, O_{3}\right) b_{O_{3}} \text { for all } O_{1}, O_{2} \in M / G
$$

where $\alpha\left(O_{1}, O_{2}, O_{3}\right)=\left|\left\{\left(m_{1}, m_{2}\right) \in O_{1} \times O_{2} \mid m_{1} \circ m_{2}=m_{3}\right\}\right|$ for some fixed $m_{3} \in$ $\mathrm{O}_{3}$. This ring (together with its distinguished basis) is called a $(M, \circ, G)$-ring.

Proof. The proof is a slight modification of the proof of Theorem (II.2) in [12]. (The semigroup ring of $M$ has to be replaced by a bigger ring.) Q.E.D.

From the inclusion reverting bijection between the (finite) Bravais groups and the Bravais manifolds one sees that (i) of (V.2) is satisfied for $\mathfrak{B}_{n}, \cap$, and (ii) follows from the Jordan-Zassenhaus Theorem. Hence the $\left(\mathfrak{B}_{n}, \cap, G L_{n}(\mathbf{Z})\right)$-ring exists. In order not to burden our description with trivial information, we will rather work with the quotient ring $\hat{\mathfrak{B}}_{n}$ of the $\left(\mathfrak{B}_{n}, \cap, G L_{n}(\mathbf{Z})\right)$-ring just described by the ideal consisting of the integral multiples of the basis vector corresponding to the empty set in $\mathfrak{B}_{n}$. Call this quotient ring $\hat{\mathfrak{B}}_{n}$ also the $\left(\mathfrak{B}_{n}, \cap, G L_{n}(\mathbf{Z})\right)$-ring.

(V.3) Proposition. The $\left(\mathfrak{B}_{n}, \cap, G L_{n}(\mathbf{Z})\right)$-ring $\hat{\mathfrak{B}}_{n}$ is isomorphic to the ring direct sum $\oplus_{l=1}^{k} \mathbf{Z}$ of $k=k(n)$ copies of $\mathbf{Z}$ where $k(n)$ is the number of $\mathbf{Z}$-classes of Bravais groups of degree $n$. More precisely, let $B_{1}, \ldots, B_{k}$ be representatives of the Z-classes of $n$-dimensional Bravais groups. Then the basis vector $b_{i}$ of $\mathfrak{B}_{n}$ corresponding to the $G L_{n}(\mathbf{Z})$-orbit of $S^{\prime}\left(B_{i}\right)$ is mapped onto $\left(\alpha_{i 1}, \ldots, \alpha_{i k}\right) \in \oplus_{i=1}^{k} \mathbf{Z}$ with $\alpha_{i j}=\mid\left\{B \leqslant B_{j} \mid B\right.$ $\left.\sim_{\mathbf{Z}} B_{i}\right\} \mid$ for $1 \leqslant i, j \leqslant k$.

Proof. The proof follows from the Galois correspondence between $\mathfrak{B}_{n} \backslash\{\phi\}$ and $\mathfrak{B}_{n}^{\prime}$ and a modification (analogous to the proof of (V.2)) of the proof of Theorem (III.1) of [12]. Q.E.D.

As an example we present the matrix $\left(\alpha_{i j}\right)$ for $\mathfrak{B}_{3}$ :

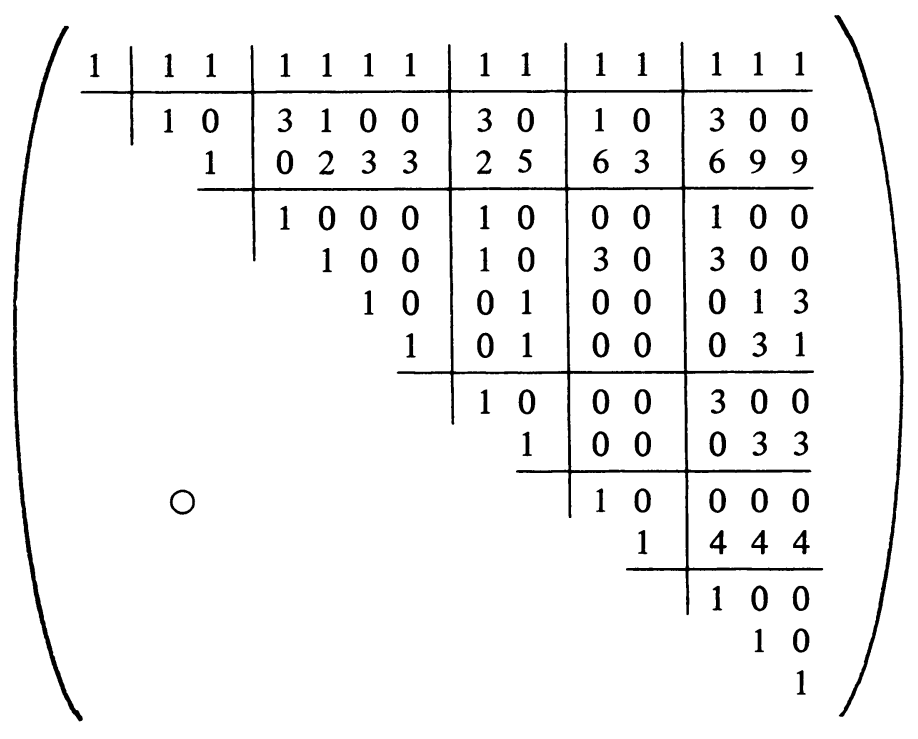


The Bravais groups (resp. manifolds) are ordered by families: $(\overline{1,1,1}),(\overline{1,1}, 1)$, $(1,1,1),\left(2_{1}, 1\right),\left(2_{2}, 1\right),(3)$ such that the almost decomposable Bravais group comes first in each reducible family. Within $(1,1,1)$ the second Bravais group is decomposable and the last two are indecomposable such that the index of the natural lattice $L$ in the associated decomposable lattice $\tilde{L}$ (see Definition (II.1)) is 2 for the third and 4 for the last. Within family (3) the ordering is given by the following sequence of Bravais manifolds: $\left\{a I_{3} \mid a>0\right\},\left\{a\left(I_{3}+J_{3}\right) \mid a>0\right\},\left\{a\left(4 I_{3}-J_{3}\right) \mid a\right.$ $>0\}$ with $I_{3}, J_{3} \in \mathbf{Z}^{3 \times 3}$ the unit- resp. all-1-matrix. (Hence in crystallographic notation the ordering is: triclinic; monoclinic $P$ and $C$; orthorombic $P, C, F$, and $I$; tetragonal $P$ and $I$; hexagonal $P$ and $R$; cubic $P, F$, and $I$.) This matrix $\left(\alpha_{i j}\right)$ stores all information on $\hat{\mathfrak{B}}_{3}$ : The rows represent the distinguished $\mathbf{Z}$-basis of $\hat{\mathfrak{B}}_{3}$ and have to be multiplied componentwise. For instance, multiplying the second and third row and writing the product again as a linear combination of the rows shows: $b_{2} b_{3}=2 b_{5}$ $+4 b_{8}$ thus giving all possible types of intersections of Bravais manifolds of different monoclinic types with multiplicities counted: e.g., a two-dimensional Bravais manifold of primitive tetragonal type can be obtained in 4 ways as intersection of four-dimensional Bravais manifolds of the two different monoclinic types. We think that the matrix $\left(\alpha_{i j}\right)$ for some higher dimensions than 3 is still in the range of computational possibilities and could support the geometric study of Bravais manifolds.

RWTH Aachen

Lehrstuhl D für Mathematik

Templergraben 64

51, Aachen, West Germany

1. H. Brown, R. Bülow, J. NeubüSER, H. WondratscheK \& H. ZasSEnhaus, Crystallographic Groups of Four-Dimensional Space, Wiley-Interscience, New York, 1978.

2. H. Brown, J. NeubüSER \& H. ZasSEnhaus, "On integral groups II; The irreducible case," Numer. Math., v. 20, 1972, pp. 22-31.

3. J. Cannon, A General Purpose Group Theory Program, Lecture Notes in Math., Vol. 372 , Springer-Verlag, Berlin and New York, 1973.

4. J. D. JaRRat, The Decomposition of Crystal Families, Report Series No. 151, Dept. of Math., Univ. of Auckland, New Zealand, 1979.

5. J. NeUBüSER, Computing Moderately Large Groups: Some Methods and Applications, SIAM-AMSProc., Vol. 4, Amer. Math. Soc., Providence, R. I., 1971.

6. J. Neubüser, W. Plesken \& H. Wondratschek, An Emendatory Discursion on Defining Crystal Systems, Proc. Conf. on Crystallographic Groups (Univ. Bielefeld, Bielefeld, 1979), Match No. 10, 1981, pp. 77-96.

7. W. PlesKen, Beiträge zur Bestimmung der endlichen irreduziblen Untergruppen von $G L(n, \mathbf{Z})$, Dissertation, RWTH Aachen, 1974.

8. W. PLESKEN, "The Bravais group and the normalizer of a reducible finite subgroup of $G L(n, \mathbf{Z})$," Comm. Algebra, v. 5, 1977, pp. 375-396.

9. W. PLESKEN, “On reducible and decomposable representations of orders," J. Reine Angew. Math., v. 297, 1978, pp. 188-210.

10. W. Plesken, Bravais Groups in Low Dimensions, Proc. Conf. on Crystallographic Groups (Univ. Bielefeld, Bielefeld, 1979), Part II, Match No. 10, 1981, pp. 97-119.

11. W. Plesken, "Applications of the theory of orders to crystallographic groups," in Integral Representations and Applications (Proceedings, Oberwolfach, Germany 1980); edited by K. W. Roggenkamp, Lecture Notes in Math., Vol. 882, Springer-Verlag, Berlin and New York, 1981, pp. 37-92. 
12. W. Plesken, “Counting with groups and rings," J. Reine Angew. Math., v. 334, 1982, pp. 40-68.

13. W. Plesken \& M. POHST, "On maximal finite irreducible subgroups of $G L(n, \mathbf{Z})$. I. The five and seven dimensional case," Math. Comp., v. 31, 1977, pp. 536-551; “II. The six dimensional case,” ibid., pp. $552-573$.

14. W. Plesken \& M. Pohst, "On maximal finite irreducible subgroups of $G L(n, \mathbf{Z})$. III. The nine dimensional case," Math. Comp., v. 34, 1980, pp. 245-258; “IV. Remarks on even dimensions with applications to $n=8$," ibid., pp. 259-275; "V. The eight dimensional case and a complete description of dimensions less than ten," ibid., pp. 277-301.

15. S. S. RySKow \& S. D. LOMAKINA, "The proof of the theorems on the maximal finite groups of integral 5 × 5-matrices," Trudy Mat. Inst. Steklov., v. 152, 1980, pp. 204-215. (Russian) 